Proceedings

\title{
Integrating Thermographic Images in A User- Friendly Platform to Support Inspection of Railway Bridges ${ }^{+}$
}

\author{
Roberto Olmi 1, Lorenzo Palombi 1, Simone Durazzani ${ }^{2}$, Daniele Poggi ${ }^{2}$, Nicolò Renzoni ${ }^{2}$, \\ Fabrizio Costantino ${ }^{2}$, Stefano Durazzani ${ }^{3}$, Gianni Frilli ${ }^{3}$ and Valentina Raimondi ${ }^{1, *}$ \\ 1 Applied Physics Institute of the National Research Councill (FAC-CNR), 50019 Sesto Fiorentino, Italy; \\ r.olmi@ifac.cnr.it (R.O.); l.palombi@ifac.cnr.it (L.P.) \\ 2 TESIFER srl, 50127 Firenze, Italy; simone.durazzani@tesifer.it (S.D.); daniele.poggi@tesifer.it (D.P.); \\ nicolo.renzoni@tesifer.it (N.R.); fabrizio.costantino@tesifer.it (F.C.) \\ 3 Durazzani srl, 50142 Firenze, Italy; territorioambiente@durazzani.it (S.D.); gianni.frilli@durazzani.it (G.F.) \\ * Correspondence: v.raimondi@ifac.cnr.it; Tel.: +39-055-5226379 \\ + Presented at the 15th International Workshop on Advanced Infrared Technology and Applications (AITA \\ 2019), Florence, Italy, 17-19 September 2019.
}

Published: 19 September 2019

\begin{abstract}
: preventive inspection of railway bridges is a key step for an effective maintenance program. The TOSCA-FI project has developed an integrated ICT platform for easy access to a wide set of data, obtained with several imaging techniques (high definition photography, photogrammetry, 3D scanning, fluorescence LIDAR and thermography) and integrated into a 3D model of the bridge. The aim is to provide a tool to support the inspection of railway bridges, often based exclusively on the visual inspection. In this paper, we present selected results obtained on masonry bridges by using thermography and their integration in the TOSCA-FI platform.
\end{abstract}

Keywords: thermography; railways; bridges; non destructive testing; ICT platform; 3D scanning; drone; diagnostics

\section{Introduction}

The built structures of the railway infrastructure, such as bridges, tunnels, etc., are inevitably prone to a progressive degradation over time, which puts at risk the efficiency and safety of use.

The durability of these structures relies on an extensive preventive inspection plan, based on which it is possible to activate an effective maintenance program. The implementation of a correct and effective inspection plan makes it possible to plan maintenance and therefore maintain the assets in efficiency, guaranteeing the safe use of the work. However, an accurate inspection plan for a large infrastructure requires many resources, both in terms of time and personnel.

Recently, the Tuscany Region has funded a project-the TOSCA-FI project-whose objective is the development of an advanced, integrated ICT tool for the diagnostics on bridges of the railway infrastructure, with a focus on cement, masonry and steel bridges. Several imaging techniques, such as high definition photography, 3D scanning, fluorescence LIDAR and thermography, were first tested in the laboratory on models specifically arranged using materials employed for the construction of railway bridges. The techniques applied in the lab were subsequently experimented in the field, on selected areas of three bridges located in the regional territory. The results were finally integrated into an ICT platform - the TOSCA-FI platform - developed in the frame of the project. The TOSCA-FI platform can be used to store and visualize thematic maps obtained by processing the data 
and to compare them over time (historical archiving), with the aim to provide a tool to support the inspection and control of railway infrastructures, which presently is mainly based on mere visual inspection. Up to now, there were only few attempts to integrate thematic maps acquired with different techniques into a unique ICT platform [1], and most of them are only limited to data integration between thermographic images and 3D models [2-5].

In this paper, we present selected results obtained on masonry bridges by using the thermographic technique [6] and their integration in the TOSCA-FI platform.

\section{Materials and Methods}

The railway bridge object of the experimental tests was a masonry-built bridge with a plaster coating. The bridge, whose reconstruction dates back to 1945-1946, is located in Debbia area, Massa, Italy. The techniques applied in the course of the measurement campaign carried out at this site were: 3D scanning, thermography, fluorescence LIDAR, high resolution photography, photogrammetry.

The bridge's 3D model was implemented by using photogrammetric images acquired with a drone. The thermographic images were acquired using a FLIR T1020 thermo-camera equipped with standard lens $\left(36 \mathrm{~mm}-\mathrm{FOV} 28^{\circ} \times 21^{\circ}\right)$ and tele-objective $\left(83.4 \mathrm{~mm}-\mathrm{FOV} 12^{\circ} \times 9^{\circ}\right)$. The fluorescence LIDAR images were obtained using an in-house developed fluorescence LIDAR [7].

\section{Thermographic Images on Masonry Bridges}

Thermographic images were acquired in several selected areas of the bridge with direct sun heating and/or in shadow. Figure 1a shows the co-registered visible image of one of the investigated areas, while Figure $1 \mathrm{~b}$ shows the relevant thermographic image. This area of the bridge was affected by plaster detaching: the areas affected by plaster detachment can be spotted in the false color-coded thermographic image as light yellow-color areas (Figure 1b). The position of the areas featuring plaster detaching in Figure1b was consistent with the observation of the surface done with grazing light in early morning (red encircled areas in Figure 1c). It is to be noted that the areas with plaster detaching could not be spotted by the naked eye unless when they were hit by grazing light.

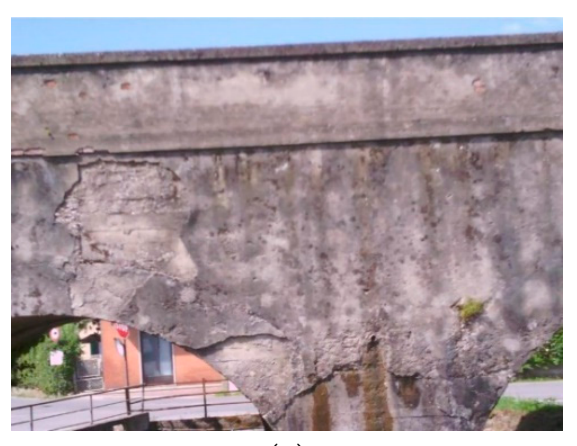

(a)

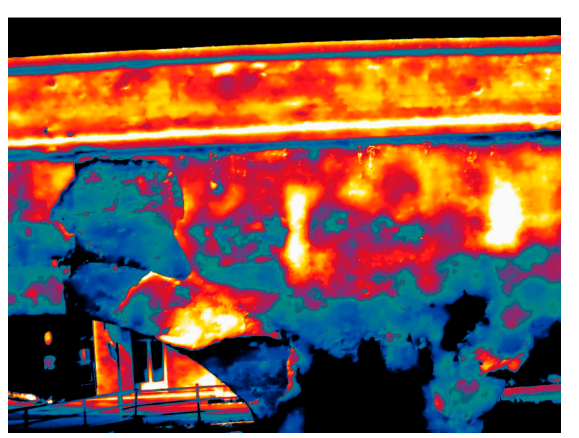

(b)

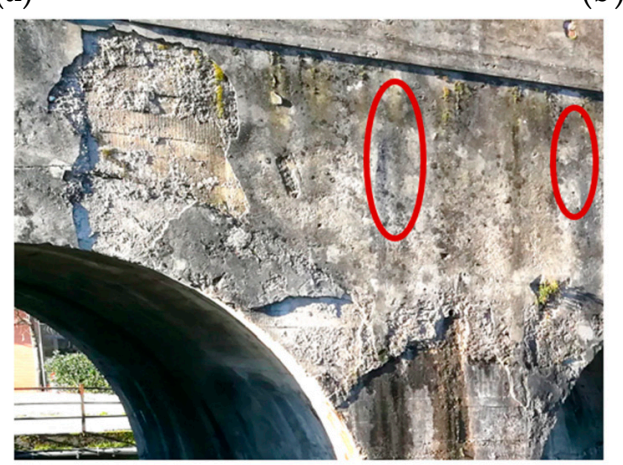

(c)

Figure 1. Thermographic acquisitions on a railway masonry bridge at Debbia (Massa, Italy): (a) Coregistered image in the visible; (b) False-color coded thermographic image; (c) Photo of the examined surface hit by grazing light. 


\section{The TOSCA-FI Platform}

The TOSCA-FI platform has been designed in order to make it possible easy access to a wide set of data and information. The database includes a map of the railway infrastructure on which it is possible to select the structure (bridge) of interest (Figure 2a); the selection of a specific bridge of the railway infrastructure makes it visible a 3D model of the bridge (Figure 2b), on which it is possible to select the areas of interest (colored areas in Figure 2b). Several thematic maps obtained by processing the data acquired with different imaging techniques can be accessed by clicking on the colored areas of interest. Figure $2 \mathrm{c}$ shows the false color-coded thermographic image superimposed to a selected area of the 3D model. On the upright of Figure 3c there is scroll bar that makes it possible to select other thematic maps, acquired on the same area yet using different techniques.

In order to integrate the thematic maps/images obtained with the different techniques on High Definition (HD) bidimensional metric digital photos (selectable from the 3D model displayed in the ICT integrated platform), we needed to develop a perspective rectification system. The latter was required to compensate the difference in perspective due to the different observation geometry of the various instruments. The image registration was obtained by applying a transformation based on at least 4 pairs of affine points.

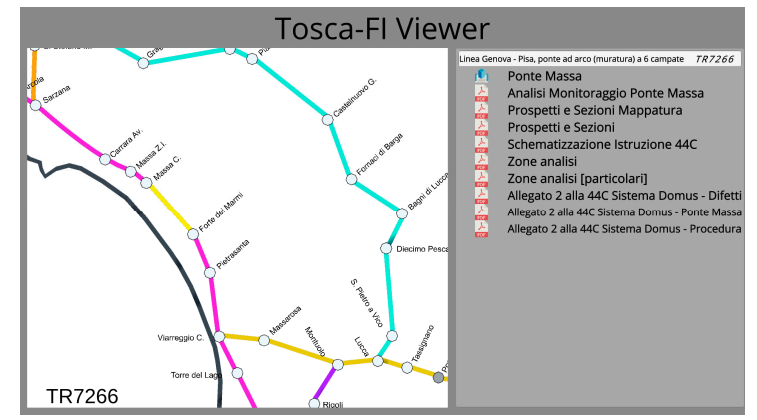

(a)

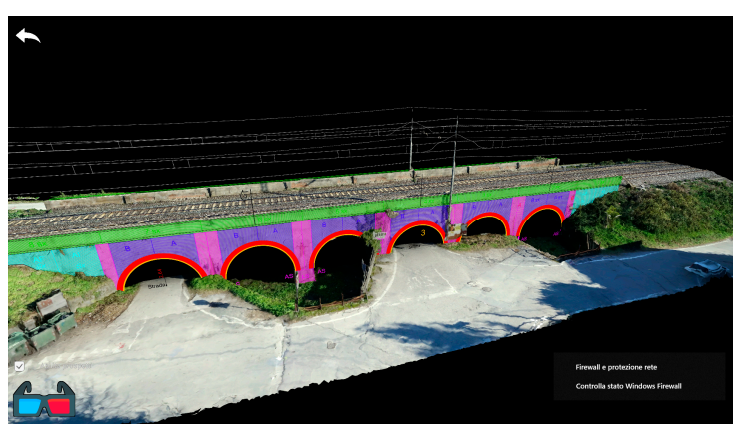

(b)

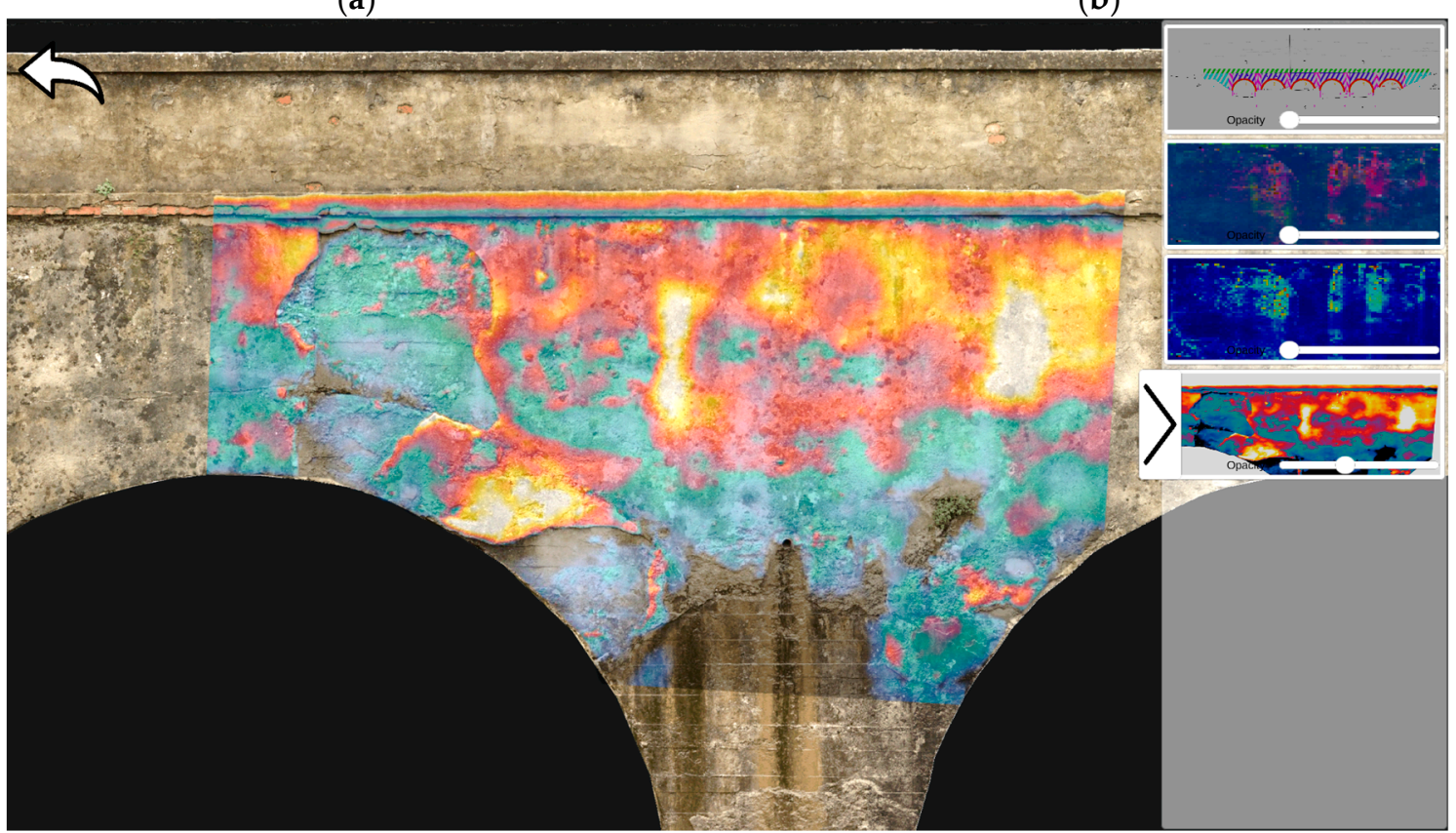

(c)

Figure 2. TOSCA-FI platform: (a) Railway infrastructure map for selecting the area (and, e.g., railway bridge) of interest; (b) 3D reconstruction of the selected structure (bridge); (c) thermographic image superimposed to the HD metric digital photos (selectable from the 3D model displayed in the ICT integrated platform). 
During laboratory tests, we used specific markers placed on the surface of the laboratory models in order to identify such points. In the real case studies (bridges), we used specific, well-identified features on the surface as affine points. It is also to be noted that, in the case of the thermographic camera, there were also available the corresponding native co-registered photos acquired with the camera to make it easier this process. In the case of the fluorescence LIDAR, the instrument was instead equipped with a laser-based reference system in the visible so that each fluorescence LIDAR dataset was completed by a set of photos with visible reference laser spots on the surface that made it possible to define with a good precision the area that was acquired.

\section{Discussion and Conclusions}

The 3D laser scanner surveys, integrated with photos, was the best starting point as a basis for the integration of thermographic images and thematic maps acquired with other techniques such fluorescence LIDAR imaging. The 3D technique provided an overall representation of the structure, both in terms of geometry and defects. The use of the drone for aerial photogrammetry has made it possible to obtain geo-localized surveys combined with high-definition images, although the precision of the point cloud is worse (some centimeters) with respect to that of the ground-based 3D scanner (some millimeters). However, the reconstruction based on drone acquisition is easier to be handled in the ICT platform, yet maintaining a very high quality image. Thus, the TOSCA-FI platform relies on the drone-based 3D reconstruction of the bridge for an overall overview of the structure, while specific areas of the bridge can be analyzed using ground-based 3D scans for an indepth analysis for example, of cracks or roto-translation of the superstructures. Thermal imaging was demonstrated to be very useful to detect some types of defects, mainly surface defects like detachments and weathered pathways on the surfaces. Thermographic images, however, are better exploited if they are complemented with photographic images. False-color coded thematic maps based on fluorescence LIDAR data were instead found useful for the detection of biofilms and patinas on the surface and the characterization of materials, providing in general information complementary to those given by the thermographic images.

In conclusion, the thermographic data and the TOSCA-FI platform can be considered as a step forward for the monitoring activity of the railway infrastructure as a support for routine inspection, although it cannot completely replace the standard procedures applied according to the ongoing regulations in the railways sector.

Author Contributions: conceptualization, Sim.D. and V.R.; software, D.P., F.C. and N.R.; thermographic measurements, R.O.; LIDAR measurements: L.P. and V.R.; 3D scanning and drone acquisition, Stef.D. and G.F.; data analysis, L.P., R.O., V.R.; writing, V.R.; funding acquisition, V.R. and Sim.D.

Funding: This research was funded by Regione Toscana grant number “CUP CIPE D18I16000250008” under the pogramme: BANDO FAR-FAS 2014 Accordo Di Programma MIUR-Regione Toscana Dgrt 1208/12 - Accordo Di Programma Quadro MIUR-MISE-Regione Toscana - Dgrt 758/2013 - Par Fas 2007-2013 - Linea D'azione 1.1.

Acknowledgments: The authors acknowledge Ferrovie Italiane S.p.A. for their support to experimental activities at the test sites.

Conflicts of Interest: The authors declare no conflict of interest.

\section{References}

1. Palombi, L.; Galeotti, M.; Massa, E.; Olmi, R.; Picollo, M.; Andreotti, A.; Bartolozzi, G.; Bini, M.; Bonaduce, I.; Cantisani, E.; et al. The use of IR-based techniques in the PRIMARTE project: an integrated approach to the diagnostics of the cultural heritage. In Proceedings of 13th International Workshop on Advanced Infrared Technology \& Applications, Pisa, Italy, 29 September 2015; pp. 313-316.

2. Cabrelles, M.; Galcerá, S.; Navarro, S.; Lerma, J.L.; Akasheh, T.; Haddad, N. Integration of 3D laser scanning, photogrammetry and thermography to record architectural monuments. In Proceedings of the 22nd International CIPA Symposium, Kyoto, Japan, 11-15 October 2009; pp. 121-124.

3. Alba, M.I.; Barazzetti, L.; Scaioni, M.; Rosina, E.; Previtali, M. Mapping Infrared Data on Terrestrial Laser Scanning 3D Models of Buildings. Remote Sens. 2011, 3, 1847-1870. 
4. Costanzo, A.; Minasi, M.; Casula, G.; Musacchio, M.; Buongiorno, M.F. Combined use of terrestrial laser scanning and IR thermography applied to a historical building. Sensors 2015, 15, 194-213.

5. González-Aguilera, D.; Lagüela, S.; Rodríguez-Gonzálvez, P.; Hernández-López, D. Image-based thermographic modeling for assessing energy efficiency of buildings façades. Energy Build. 2013, 65, 29-36.

6. Olmi R.; Inglese G. Measurement of the external parameters in quantitative active thermography. Meas. Sci. Technol. 2017, 28, 105403.

7. Palombi, L.; Alderighi, D.; Cecchi, G.; Raimondi, V.; Toci, G.; Lognoli, D. A fluorescence LIDAR sensor for hyper-spectral time-resolved remote sensing and mapping. Opt. Express 2013, 21, 14736-14746.

(C) 2019 by the authors. Licensee MDPI, Basel, Switzerland. This article is an open access article distributed under the terms and conditions of the Creative Commons Attribution (CC BY) license (http://creativecommons.org/licenses/by/4.0/). 